\title{
AN INTEGRATED ADAPTIVE MODEL FOR OVERHEATING RISK PREDICTION
}

\author{
Darren Robinson1 and Frédéric Haldi1
}

1Solar Energy and Building Physics Laboratory (LESO-PB), Ecole Polytechnique Fédérale de Lausanne (EPFL), CH-1015 Lausanne, Switzerland.

\begin{abstract}
$\underline{\text { ABSTRACT }}$
Based on results from a field survey campaign, this paper describes three new developments which have been integrated to provide for a comprehensive basis for the evaluation of overheating risk in offices. Firstly, a set of logistic regression equations have been derived to predict the probability of office occupants' adaptation of personal and environmental characteristics. Secondly, empirical adaptive increments (offsets in comfort temperature) have been derived for each of these modes of adaptation. Thirdly, these adaptive increments are used to derive adapted degree-days of overheating stimuli for input to a new model to predict overheating risk. Based on analogy between the charging and discharging of humans' tolerance to overheating stimuli and that of charge in an electrical capacitor, this analytical model uses empirical coefficients to tune its (dis)charging time constants to a given population and situation. This paper introduces these developments and discusses scope for their further development.
\end{abstract}

\section{KEYWORDS}

Thermal comfort, overheating risk, adaptive actions, empirical adaptive increments, integration.

\section{INTRODUCTION}

In the summer of 2006 a detailed thermal comfort field survey campaign was conducted in several Swiss offices. This field survey was motivated by the desire to better understand the causes for overheating in offices and to develop a basis for the predicting the probability of its occurrence. In particular we wished to understand whether overheating was due to an accumulation of overheating stimuli or to a single heat stress event.

We also wished to know whether and to what extent occupants are forgiving of these overheating stimuli when they have exercised opportunities to adapt their personal and/or environmental characteristics.

Finally, if adaptive actions do influence overheating risk then, for this to be useful, some basis for predicting the probability that these actions will take place should be provided for. This latter would then provide an input to a building simulation program which would in turn predict the corresponding physical responses of these adaptive actions.

\section{FIELD SURVEY METHODOLOGY}

During the summer of 2006 a field survey was conducted in eight non air-conditioned office buildings, each located within a $50 \mathrm{~km}$ radius of Lausanne (latitude $46.5^{\circ} \mathrm{N}$, longitude $6.7^{\circ} \mathrm{E}$ ) Switzerland. Their selection was based on a desire for reasonable diversity in terms of their design concept and the adaptive opportunities available to occupants.

For each building, volunteers were asked to complete a short electronic questionnaire which was installed on their PC. This questionnaire (Figure 1), which appeared at regular participant-defined intervals, asked for evaluations of:

- Clothing and activity level.

- Thermal satisfaction and preference.

- Adaptive opportunities exercised.

The purpose of this dialogue box was to produce timeseries data regarding participants' adaptive actions and their evolving perception of the parameter(s) under examination.

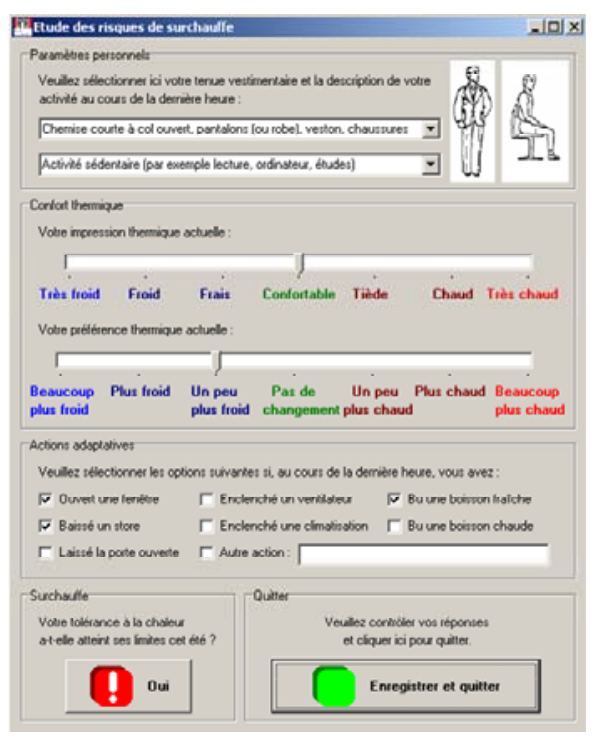

Figure 1 Longitudinal e-questionnaire

Participants were also asked to press an 'overheated' button once they felt that their thermal tolerance had been surpassed. To ensure that we would obtain unambiguous information participants were asked to select this option one time only. After confirming their choice, this prompted a further dialogue box (Figure 2) which requested participants to describe the 
circumstances that led to this transition in tolerance (i.e. from the space having been thermally acceptable to now having overheated). More specifically whether this was caused by excessive temperatures during the moment in question, throughout the day in question or throughout several prior days. Participants were also asked to remark whether this overheating was influenced by excess solar gain, a lack of control options or to offer alternative explanations.

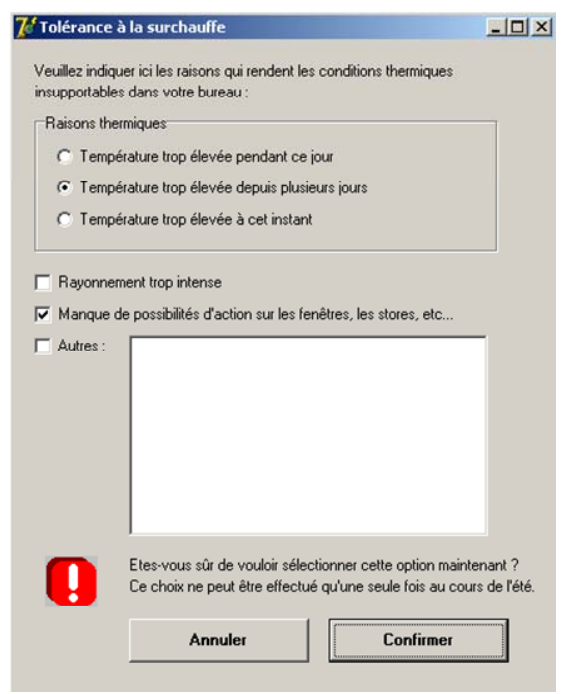

Figure 2 Dialogue box for explanation of cause of overheating

Occupants' responses to the questionnaire were appended to a local data file, generally on a twohourly basis (i.e. most participants completed the questionnaires four times per day). In parallel, measurements were recorded at $45 \mathrm{~min}$ intervals from calibrated solar-shielded temperature sensors, installed in close proximity to each participant's workstation. Finally, at the end of the study, local simultaneous climate data was obtained from the Swiss Federal Office of the Environment.

In total there were 60 participants in this study, who produced a total of 5908 responses (i.e. each participant completed the questionnaire an average 98 times) for the period 13 June to 27 September 2006.

\section{OVERHEATING RISK}

Of the sixty participants only twenty two reported that their thermal tolerance had been exceeded. Of these only $18 \%$ indicated that this was caused by excess temperature at the moment in question, whereas $27 \%$ and $55 \%$ indicated that this was due to excess temperature during the day in question and during the past several days respectively.

This result tends to confirm that occupants are tolerant of occasional departures from comfort; or more specifically that overheating is due to an accumulation of heat stress events rather than a single event (which we may regard as a special case of an accumulation of heat stresses over a very short period of time).
Following from this rationale that it is an accumulation of stimuli that leads us to consider a space as having overheated, the principal hypothesis of our model is that (the storage of) human tolerance to overheating stimuli can be considered as equivalent to the storage of electrical charge in a capacitor. By this it is meant that during a series of particularly warm days this overheating tolerance is discharged. If this is followed by a cooler period then this tolerance is recharged. An extreme example of this is a recharging of our tolerance during winter-time in readiness for discharging during the following summer. If tolerance $T \in[0,1]$ then the probability of overheating $P_{O H}=1-T$.

Now, let us consider the well-known situation of a circuit including a capacitance $C$ and a resistance $R$, receiving a constant tension $U_{0}$. The tensions in these components are linked by the equation $U_{0}=U_{R}+U_{C}$, which leads to the differential equation: $\quad R C \frac{d U_{C}}{d t}(t)+U_{C}(t)=U_{0} \quad$. Setting $U_{C 0}=U_{C}(t=0)$, we obtain the well-known solution for the discharging of the capacitor: $U_{C}(t)=U_{C_{0}} \exp (-t / R C)$ and for its corresponding charging we have: $U_{C}(t)=U_{0}(1-\exp (-t / R C))+U_{C_{0}} \exp (-t / R C)$; where the product $R C$ is a (dis)charging time constant $\tau$. In the context of our overheating model, we shall set $U_{0}=1$ and interpret $U_{C}(t)$ as the probability of having overheated $P_{\mathrm{OH}}(t)$ due to an accumulation of heat stimuli and $U_{R}(t)$ as the tolerance to these stimuli, so that $U_{R}(t)+U_{C}(t)=1$ for any time $t$.

We assume that the heat stimuli may be represented by the excess of some reference temperature $\theta^{*}$, say $25^{\circ} \mathrm{C}$. We therefore replace the reciprocal of our time constant $1 / \tau$ with the time-varying expression $\alpha\left(\theta(t)-\theta^{*}\right)$ in the charge expression, given a constant $\alpha$, and with $\beta\left(\theta^{*}-\theta(t)\right)$ in the discharge expression.

Considering now the time interval $t-\Delta t, t$ we have the following expression for the probability of overheating as a function of $P_{O H}(t-\Delta t)$ which, whilst charging, is:

$$
P_{O H}(t)=1-e^{-\alpha\left(\theta(t)-\theta^{*}\right) t} \cdot\left(1-P_{O H}(t-\Delta t)\right)
$$

and during discharging is:

$$
P_{O H}(t)=e^{-\beta\left(\theta^{*}-\theta(t)\right) t} \cdot\left(P_{O H}(t-\Delta t)\right)
$$

with $\alpha, \beta$ being parameters to determine empirically.

Let us now take $n$ consecutive periods of charge $t_{0}, t_{0}+\Delta t . . t_{n}-\Delta t, t_{n}$ during the period $t_{0}, t_{n}$. Using 
Eq. [1a], inserting a similar expression for $P_{O H}(t-\Delta t)$ and solving recurrently until $t_{0}$, we have for charging:

$$
\begin{aligned}
& P_{O H}\left(t_{n}\right)=1-\exp \left(-\alpha \sum_{i=0}^{n-1}\left[\left(\theta(t-i \Delta t)-\theta^{*}\right)(t-i \Delta t)\right]\right) \\
& \cdot\left(1-P_{O H}\left(t_{0}\right)\right)
\end{aligned}
$$

Now, if we define degree-hours (DH) of heat stimuli above our reference temperature during the period $t_{i}, t_{j}$ as $D H_{t i, t j}=\left(\theta\left(t_{i}\right)-\theta^{*}\right) \cdot\left(t_{j}-t_{i}\right)$ then we obtain the following for charging:

$$
P_{O H}\left(t_{n}\right)=1-\exp \left(-\alpha \sum_{i=1}^{n} D H_{t_{0}, t_{i}}\right) \cdot\left(1-P_{O H}\left(t_{0}\right)\right)
$$

And for discharging during $n$ consecutive periods $t_{0}, t_{0}+\Delta t, \ldots, t_{n}-\Delta t, t_{n}$ :

$$
P_{O H}\left(t_{n}\right)=\exp \left(-\beta \sum_{i=1}^{n} D H^{*} t_{0}, t_{i}\right) \cdot P_{O H}\left(t_{0}\right)
$$

Where $P_{O H}\left(t_{0}\right)$ is either zero at the start of a simulation or refers to the probability of overheating at the time of transition from charging to discharging (or vice versa) of our human capacitor. $D H_{t i, t j}^{*}=\left(\theta^{*}-\theta\left(t_{i}\right)\right) \cdot\left(t_{j}-t_{i}\right)$. Thus we are able to model a temporal evolution of the probability of overheating within the limits $[0,1]$ during an arbitrary time period.

Now, during the warmest period of our field survey campaign in which all overheating events were logged, no internal air temperature below $25^{\circ} \mathrm{C}$ was recorded during working hours. The period was thus a continuously charging period of overheating probability (or inversely a discharging of tolerance). Under such (continuously charging) circumstances we are able to simplify Eq. 3a somewhat, so that:

$$
P_{O H}(t)=1-\exp \left(-\alpha^{\prime} D H_{t 0, t}\right)
$$

where $\alpha^{\prime}=\alpha \Delta t$. Determining $\alpha^{\prime}$ is then performed by the fitting of data using the regression equation $\log \left(1-P_{O H}(t)\right)=-\alpha^{\prime} D H_{t 0, t}$ where $P_{O H}$ is the observed cumulative probability of overheating. Performing this regression on our data gives a value of $\alpha^{\prime}=4.75 \cdot 10^{-4}\left[\mathrm{~K}^{-1} \mathrm{~h}^{-1}\right]$ with good agreement $\left(\mathrm{r}^{2}=\right.$ 0.98 ), albeit perhaps based on a limited range of $0<$ $\left(\mathrm{DH}>25^{\circ} \mathrm{C}\right)<1200$.

The corresponding predicted probability of overheating using this simplified form of the model (i.e. for continuous charging) is compared with the measurement results in Figure 3.

Note that this new model predicts the probability that a population will perceive a given space to have overheated, but we may also interpret this as the proportion of a given population that will perceive a space to have overheated.
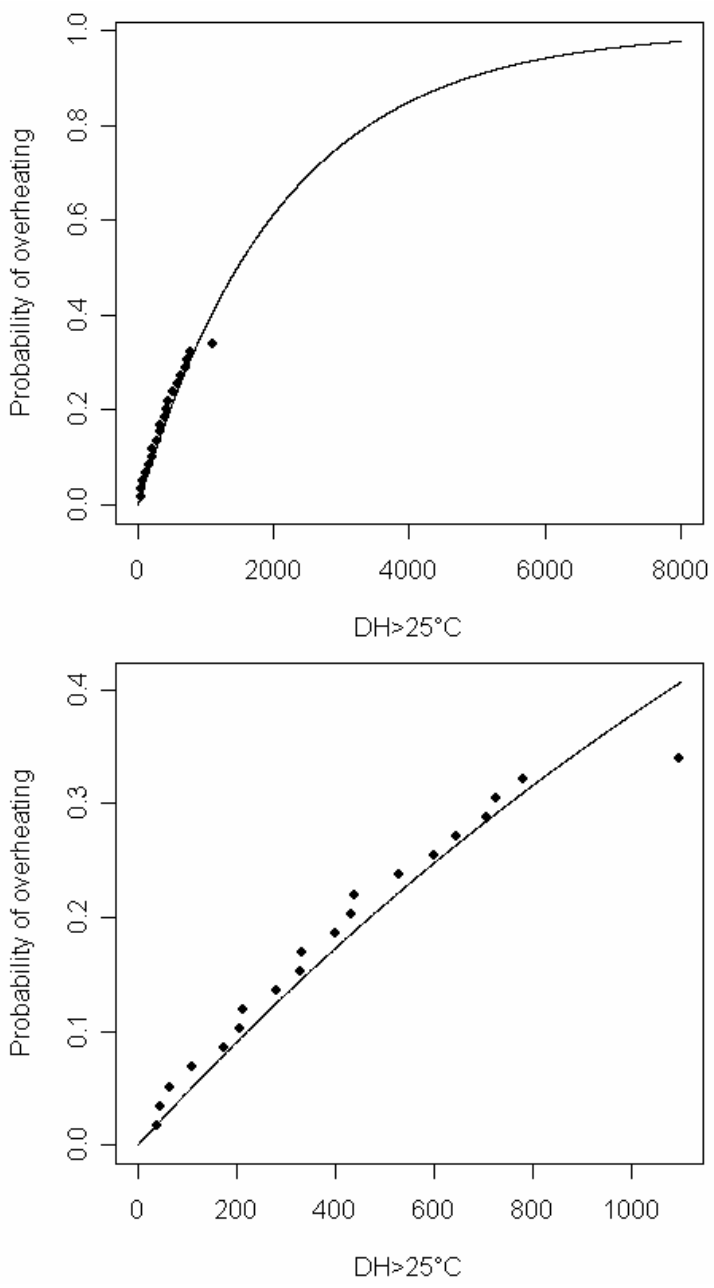

Figure 3.Predicted (solid line) and measured (solid diamonds) overheating probability as a function of $D H>25^{\circ} \mathrm{C}$ in the probability range [0,1] (top) and focussing on the range [0,0.4] (bottom).

Now, to test our model thoroughly we would ideally have data spanning the entire range $P_{O H}[0,1]$, but unfortunately the summer of 2006 was not sufficiently warm to provide us with these data. Nevertheless we know that building designers seek to ensure the comfort of the majority of a building population, so that current thermal comfort standards target a PPD of $\leq 20 \%$. We may therefore suggest that our model is valid within the range of practical application [i.e. it compares well with measurements in the range $0 \leq P_{\mathrm{OH}} \leq 0.2$ that is of interest to building designers].

See Robinson and Haldi (2007) for further discussion.

Although we were unable to obtain data with which to calibrate $\beta$ (for this we would need at least two warm periods interrupted by a cool period), we suspect that $\beta » \alpha$ (perhaps of the order $\beta \approx 10 \alpha^{\prime}$ ). This is because we suppose that occupants are psychologically more sensitive to the thermal relief that they feel during a cool period which follows a warm period in which their tolerance has been gradually discharged. 


\section{ADAPTIVE ACTIONS}

We suppose that people adapt their personal and/or environmental characteristics to suppress or diminish discomforting stimuli. This follows from the adaptive principle stated by Baker and Standeven (1996) that: "If a change in the thermal environment occurs, such as to produce discomfort, people react in ways which tend to restore their comfort".

In particular we focus on the influence of thermal stimuli on occupants' interactions with windows, blinds, fans and doors, and their consumption of cold drinks. More specifically, and inspired by the work of Nicol (2001) and Rijal et al (2007), we attempt to infer a distribution for the probability of occupants' adaptive actions as a function of indoor and outdoor temperature.

For each unit temperature $\theta_{k}$, the ratio between the occurrences of control action $N\left(\theta_{k}\right)$ and the total amount of occurrences of this temperature $N_{t o t}\left(\theta_{k}\right)$ is computed, giving an empirical probability of adaptive action at temperature $\theta_{k}$. The error associated with each of these action probabilities at each temperature $\theta_{k}$ is obtained by dividing $\sigma_{N}\left(\theta_{k}\right)$, i.e. the standard deviation of $N\left(\theta_{k}\right)$, by the number of occurrences $N_{\text {tot }}\left(\theta_{k}\right)$ for a given temperature $\theta_{k}$. In order to infer a probability distribution for the whole range of temperatures, a statistical method already used for such purposes in Nicol (2001) and Rijal et al (2007) is logistic regression. The proposed probability distribution $p(\theta)$ then follows the relationship: $\log \left(\frac{p(\theta)}{1-p(\theta)}\right)=a \theta+b$. The probability distribution is then given by the logit function: $p(\theta)=\frac{\exp (a \theta+b)}{1+\exp (a \theta+b)}$.

We have performed this regression analysis for the adaptive control options opening windows, lowering blinds, switching on fans, opening doors and consuming cold drinks. In all cases we find that these actions are significantly better described by indoor rather than outdoor temperature (Haldi and Robinson, 2007). We therefore present here only the results relating to indoor temperature (Table 1). Although by way of illustration we present the regression data and fitted equations for window openings for both indoor and outdoor temperature (Figure 4).

Table 1 Logit function parameters for $p\left(\theta_{i}\right)$

\begin{tabular}{|l|c|c|c|}
\hline \multicolumn{1}{|c|}{ Control } & $\mathbf{a}$ & $\mathbf{b}$ & $\mathbf{r}^{\mathbf{2}}$ \\
\hline Windows & $0.224 \pm 0.027$ & $-5.77 \pm 0.68$ & 0.86 \\
\hline Blinds & $0.416 \pm 0.040$ & $-11.17 \pm 1.01$ & 0.91 \\
\hline Fans & $0.828 \pm 0.074$ & $-22.88 \pm 1.87$ & 0.93 \\
\hline Doors & $0.342 \pm 0.050$ & $-8.45 \pm 1.22$ & 0.81 \\
\hline Cold drinks & $0.227 \pm 0.019$ & $-6.54 \pm 0.48$ & 0.92 \\
\hline
\end{tabular}

It is also interesting to determine the temperature which corresponds to the $50^{\text {th }}$ percentile of action probability. This characteristic temperature $\theta_{50}$, which is simply $-b / a$, indicates the following relative sequence of interactions: doors $\left(24.68^{\circ} \mathrm{C}\right)$, windows $\left(25.77^{\circ} \mathrm{C}\right)$, blinds $\left(26.85^{\circ} \mathrm{C}\right)$, fans $\left(27.64^{\circ} \mathrm{C}\right)$, and drinks $\left(28.75^{\circ} \mathrm{C}\right)$.

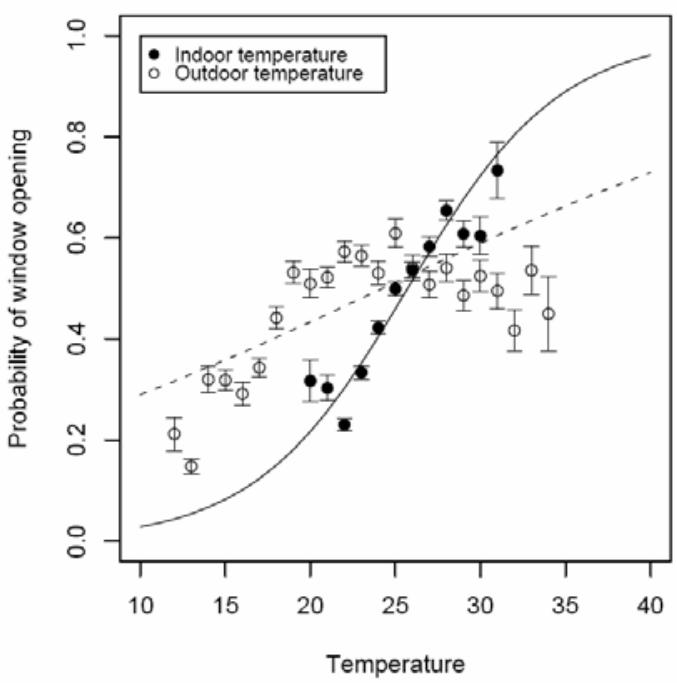

Figure 4 Window opening probability as a function of indoor and outdoor temperature

\section{EMPIRICAL ADAPTIVE INCREMENTS}

In addition to predicting the probability of exercising a given adaptive control action, we are also able to determine its effectiveness. For this we determine the difference in median temperature for "neutral" thermal sensation votes with and without having exercised a given adpative action. This is equivalent to the notion of adaptive increments proposed by Baker and Standeven (1996). In Figure 5 for example we see that for all categories of reported thermal sensation the median temperature is higher when windows have been opened. In other words occupants are tolerant of warmer internal conditions when windows have been opened. This trend is followed for all control actions.

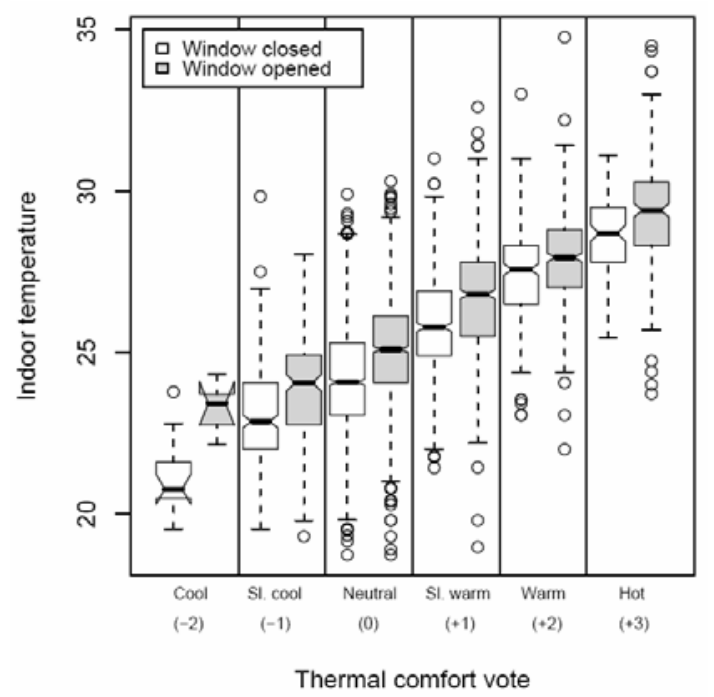

Figure 5 Influence of window opening on temperature distribution 
We have two sets of results for these empirical adaptive increments: (i) those generated for cases when the control action in question has or has not been exercised, but others may have been; and (ii) those in which this is exclusively the case (i.e. the data has been filtered to cases of no control action [without] and exclusively the control action in question [with]).

Although the latter case relates to a more rigorous definition of our adaptive increments, we retain the former for interest; as their statistical significance is higher (uncertainties are rather low).

The corresponding results are presented in tables 2 and 3 below. Note that table 3, which relates to exclusive adaptive actions, also contains results from known conjugations of control action for which we have a reasonable number of occurrences.

Table 2 Reported comfort temperatures and increments for non-exclusive control actions

\begin{tabular}{|l|c|c|c|c|c|}
\hline Control & $\begin{array}{c}\text { Without } \\
\left({ }^{\mathbf{0}} \mathbf{C}\right)\end{array}$ & $\begin{array}{c}\text { With } \\
\left({ }^{\mathbf{0}} \mathbf{C}\right)\end{array}$ & $\begin{array}{c}\text { Increment } \\
\left(\mathbf{(}^{\mathbf{0}} \mathbf{C}\right)\end{array}$ & $\begin{array}{c}\text { Occur } \\
(\mathbf{w} / \mathbf{0})\end{array}$ & $\begin{array}{c}\text { Occur } \\
(\mathbf{w}) .\end{array}$ \\
\hline Windows & $24.08 \pm .09$ & $25.09 \pm .08$ & $1.01 \pm .17$ & 1565 & 1502 \\
\hline Blinds & $24.40 \pm .07$ & $25.43 \pm .17$ & $1.03 \pm .24$ & 2490 & 577 \\
\hline Fans & $24.40 \pm .07$ & $26.49 \pm .23$ & $2.09 \pm .30$ & 2900 & 167 \\
\hline Doors & $24.40 \pm .08$ & $24.74 \pm .10$ & $0.34 \pm .18$ & 1737 & 1330 \\
\hline Cold drinks & $24.40 \pm .08$ & $24.90 \pm .13$ & $0.50 \pm .21$ & 2275 & 792 \\
\hline
\end{tabular}

Table 3 Reported comfort temperatures and increments for exclusive control actions and their conjugates

\begin{tabular}{|l|c|c|c|c|}
\hline \multicolumn{1}{|c|}{ Control } & $\begin{array}{c}\text { Tcomf } \\
\left({ }^{\mathbf{}} \mathbf{C}\right)\end{array}$ & Occur & $\begin{array}{c}\text { Increment } \\
\left({ }^{\mathbf{}} \mathbf{C}\right)\end{array}$ & $\begin{array}{c}\text { Interaction } \\
\text { constant, } \boldsymbol{\alpha}\end{array}$ \\
\hline None & $23.90 \pm .11$ & 779 & - & - \\
\hline Windows (W) & $24.62 \pm .15$ & 349 & $0.72 \pm .26$ & - \\
\hline Blinds (B) & $24.30 \pm .58$ & 28 & $0.40 \pm .69$ & - \\
\hline Fans (F) & $25.10 \pm .67$ & 16 & $1.20 \pm .78$ & - \\
\hline Doors (D) & $24.14 \pm .17$ & 339 & $0.24 \pm .28$ & - \\
\hline $\begin{array}{l}\text { Cold drinks } \\
\text { (CD) }\end{array}$ & $24.41 \pm .26$ & 204 & $0.51 \pm .38$ & - \\
\hline $\mathrm{W}+\mathrm{B}$ & $25.58 \pm .38$ & 83 & $1.68 \pm .49$ & $1.50 \pm 1.71$ \\
\hline $\mathrm{W}+\mathrm{D}$ & $24.74 \pm .18$ & 301 & $0.84 \pm .30$ & $0.88 \pm 0.80$ \\
\hline $\mathrm{W}+\mathrm{CD}$ & $24.93 \pm .24$ & 189 & $1.03 \pm .35$ & $0.84 \pm 0.72$ \\
\hline $\mathrm{B}+\mathrm{D}$ & $24.68 \pm .33$ & 49 & $0.78 \pm .44$ & $1.22 \pm 2.54$ \\
\hline $\mathrm{D}+\mathrm{CD}$ & $23.72 \pm .37$ & 103 & $-0.18 \pm .48$ & $-0.24 \pm 0.43$ \\
\hline $\mathrm{W}+\mathrm{B}+\mathrm{D}$ & $25.43 \pm .30$ & 221 & $1.53 \pm .41$ & $0.94 \pm 0.95$ \\
\hline $\mathrm{W}+\mathrm{B}+\mathrm{CD}$ & $24.40 \pm .45$ & 45 & $0.50 \pm .56$ & $0.31 \pm 0.59$ \\
\hline $\mathrm{W}+\mathrm{D}+\mathrm{CD}$ & $25.09 \pm .18$ & 153 & $1.19 \pm .29$ & $0.81 \pm 0.70$ \\
\hline $\mathrm{W}+\mathrm{F}+\mathrm{D}$ & $26.59 \pm .70$ & 25 & $2.69 \pm .82$ & $1.25 \pm 1.14$ \\
\hline $\mathrm{W}+\mathrm{B}+\mathrm{F}+\mathrm{D}$ & $26.78 \pm .37$ & 60 & $2.88 \pm .49$ & $1.13 \pm 1.07$ \\
\hline $\mathrm{W}+\mathrm{B}+\mathrm{D}+\mathrm{CD}$ & $25.75 \pm .60$ & 41 & $1.85 \pm .72$ & $0.99 \pm 1.24$ \\
\hline
\end{tabular}

For some controls, when used simultaneously, increments in comfort temperature tend to be accentuated while for other conjugations they tend to be dampened. In other words conjugations of controls do not yield simply (linearly) additive increments.

Let us define the joint increments $D_{i j}$ of two controls providing individually the increments $D_{i}$ and $D_{j}$, we can set $D_{i j}=\alpha_{i j}\left(D_{i}+D_{j}\right)$, where $\alpha_{i j}$ is an interaction term. When $\alpha_{i j}>1$, an extra increment appears due to constructive interactions between controls, while when $\alpha_{i j}<1$, controls do not fully interact constructively. Extending this definition, it is also possible to evaluate three-term and four-term interaction constants. Note that for this we use results that relate exclusively to these control actions (where as the results above may have also included other actions), so that the precision of many of the associated interaction constants is dampened by statistical uncertainties in the offsets.

\section{MODEL INTEGRATION}

Until now we have presented a set of regression equations for predicting the probability of adaptive actions. We have also calculated the corresponding influences on occupants' comfort temperature and we have derived a model for the prediction of overheating risk. But the presented implementation of this model is not yet sensitive to these empirical adaptive increments - it uses raw measured/calculated degreehours of overheating stimuli, with no distinction as to whether adaptive actions have taken place or what their effects have been.

To resolve this we therefore re-calibrate our coefficients $\alpha, \beta$ using our field survey data, based on re-calculation of $D H_{t 0, t}$ (i.e. we reduce these degreehours according to the adaptive actions exercised). In our simplified case of continuous discharging of overheating tolerance we once again make use of the expression $\quad \alpha^{\prime}=-\log \left(1-P_{O H}(t)\right) / D H_{t 0, t} \quad$. The resultant non-adaptive model then becomes an adaptive one when the measured/simulated thermal input $\mathrm{DH}_{t 0, t}$ to eq. [4] has been adjusted according to the adaptive actions taken, so that for the charging case $D H_{t i, t j}=\left(\left[\theta\left(t_{i}\right)-\Delta \theta\right]-\theta^{*}\right) \cdot\left(t_{j}-t_{i}\right)$ where $\Delta \theta$ is the corresponding adaptive increment, whether this be due to single or a conjugate of actions.

This integrated model then has the form (figure 6):

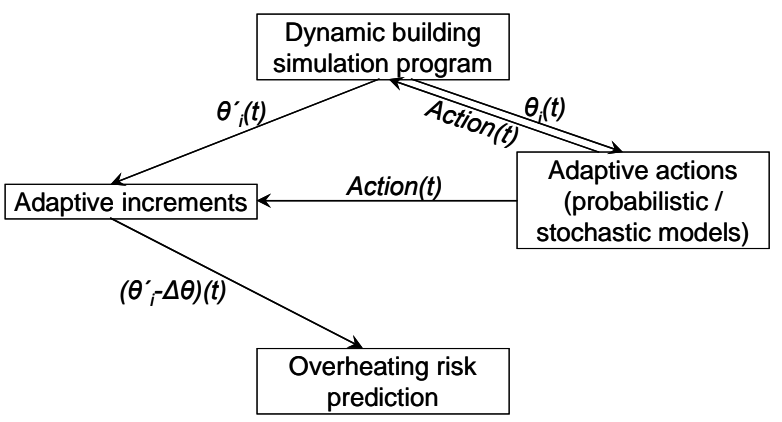

Figure 6 Schematic view of integrated model

Note that $\theta_{i}^{\prime}$ in Figure 6 corresponds to a converged temperature $\theta_{i}$ following from iterations between 
control action and the building energy balance equations.

\section{MODEL CRITIQUE}

There are many weaknesses to this new integrated model for overheating risk prediction. For example:

We currently only consider responses to indoor air temperature rather than a more comprehensive measure such as operative temperature.

- The overheating model has also been calibrated to exclusively temperate climate conditions. Its relevance to e.g. warm humid climates may be questionable.

- Also due to field survey constraints, we have not been able to calibrate the charge of tolerance to overheating stimuli $\beta$.

- The probabilistic models of adaptive actions should consider non-thermal stimuli, e.g. visual for blind use and olfactory for window openings. In this the closing of windows and raising of blinds (and switching off of fans) should also be considered; likewise the prioritisation of different control actions.

- Certain of the suggested adaptive increments suffer from rather high uncertainties.

Nevertheless, this new integrated model does represent a promising direction for the development of a complete model for predicting occupants' adaptations of their personal and environmental characteristics and the associated consequences for their overall (long term) satisfaction with the indoor thermal environment. This (overheating view) is, after all, the key test for whether a space is generally acceptable or not.

\section{CONCLUSIONS}

A new integrated model for the prediction of overheating risk in offices in temperate climates has been proposed. This model predicts:

- The probability with which occupants will adapt their personal and environmental characteristics in response to thermal stimuli.

- The effects of adaptive actions, when exercised, on occupants' comfort temperature.

- The probability of a population perceiving an environment to have overheated (or alternatively the probable proportion of a population to perceive this) in response to the above.

Finally it is interesting to note that, due to the symmetric nature of the problem, the model for predicting overheating risk may in principle also be applied for predicting underheating risk.
There are however numerous weaknesses in this work. Nevertheless, this does represent a promising step towards the completion of a fully comprehensive model for predicting occupants' interactions and satisfaction, both short and long term, with the indoor environment.

\section{ACKNOWLEDGEMENTS}

The financial support for this work from the Swiss National Science Foundation, through National Research Programme 54 Sustainable Development of the Built Environment, is gratefully acknowledged. Many thanks also to Liz Azzi for help with the field surveys to David Lindelöf for advice on statistical analysis and to Claude-Alain Roulet for his boundless enthusiasm and helpful advice.

\section{REFERENCES}

Baker, N., Standevan, M. (1996), Thermal comfort for free-running buildings, Energy and Buildings 23(3) 175-182, 1996.

Haldi, F., Robinson, D. (2007), On the behaviour and adaptation of office occupants, Energy and Buildings, (submitted).

Nicol, J. F. (2001), Characterising occupant behaviour in buildings: towards a stochastic model of occupant use of windows, lights, blinds, heaters and fans, Proc. 7th Int IBPSA Conf, Rio, p10731078.

Rijal, H.P. Tuohy, P. Humphreys, M.A. Nicol, J.F. Samuel, A. Clarke, J.A (2007), Using results from field surveys to predict the effect of open windows on thermal comfort and energy use in buildings, Energy and Buildings 39(7) 823-836, 2007.

Robinson, D., Haldi, F. (2007), Model to predict overheating risk based on an electrical capacitor analogy, Energy and Buildings, (submitted). 